\title{
Viscoelasticity and microstructure of non-ionic microemulsions
}

\author{
A. Eshuis and J. Mellema \\ Rheology Group, Department of Applied Physics, Twente University of Technology, Enschede, The Netherlands
}

\begin{abstract}
Non-ionic microemulsions were investigated by viscoelastic measurements in the $\mathrm{kHz}$ region. We found that in some parts of the phase diagram our systems consisted of a dispersion of spherical oil doplets, stabilized by a non-ionic surfactant, in a continuous phase of almost pure water. Because of the simplicity of the system used it was relatively easy to interpret our measurements in terms of two rheological models developed by Oldroyd. Using these models, we could calculate the interfacial tension between the continuous and the dispersed phase. In other parts of the phase diagram, however, our results indicate the presence of a fluctuating network of oil-swollen cylindrical micelles.
\end{abstract}

Key words: Visco-elasticity, non-ionic microemulsion, interfacial tension, relaxation time, oil-swollen micelles.

\section{Introduction}

Although considerable research activity is devoted to microemulsions nowadays, their viscoelatic properties are rarely mentioned. Nevertheless, rheological measurements may significantly increase the understanding of these systems, since, up to date, they offer the only possibility of determining the interfacial tension between the dispersed and the continuous phase. This particular parameter is very important because it influences the stability and various other properties of these systems.

According to a recent definition, a microemulsion should be a thermodynamically stable and optically translucent system [1]. Although this definition includes no requirement about the microstructure, it is often assumed that a microemulsion consists of small spherical doplets of one liquid dispersed in another one. For the time being, however, other possibilities, like rods, lamellae, or even bicontinuous structures, cannot be ruled out. We just know that the correlation length of the structure must be small compared to optical wavelengths so that these systems remain optically translucent. This, in its turn, implies that the interfacial area between the two liquids is very large. In view of the required thermodynamic stability this suggests that the interfacial tension is very low.

In this paper non-ionic microemulsions $n$-bexane/ water/polyoxyetbylene (6.8) nonylphenol are investigated. It will appear that in a certain temperature and composition range these microemulsions contain almost spherical droplets indeed. The viscoelasticity in this region is rather well described by two models developed by Oldroyd about thirty years ago $[2,3]$. The measurements allow determination of various properties of the interfacial layer.

In other temperature-composition regions, however, an interpretation in terms of an individualdroplet system is not possible. The high values of the shear-storage modulus rather suggest the presence of some kind of fluctuating network, the rigidity of which strongly increases with temperature.

\section{Theory}

\section{Linear viscoelasticity [4]}

It is a well-known fact that purely viscous liquids behave according to Newton's law:

$$
\tau=\eta \dot{\varepsilon}
$$

and purely elatic solids to Hooke's law :

$$
\tau=G \varepsilon,
$$

in which $\tau$ is the applied shear stress, $\varepsilon$ the shear, and $\dot{\varepsilon}$ the shear rate. For materials that behave linearly the viscosity $\eta$ and the shear modulus $G$ are independent of $\dot{\varepsilon}$ and $\varepsilon$, respectively. The difference in behaviour is very clearly demonstrated in oscillatory measurements. For viscous materials there is a phase difference of $90^{\circ}$ between force and deformation, whereas for elastic materials force and deformation are always exactly in phase. 
Many materials do not belong to one of these categories. They show a combination of viscous and elastic behaviour. For sufficiently low $\varepsilon$ or $\dot{\varepsilon}$ they show linear viscoelastic behaviour. This means that they can be characterized by the complex viscosity $\eta^{*}$, defined as

$$
\eta^{*} \equiv \frac{\hat{\tau}}{\hat{\dot{\varepsilon}}}
$$

or, alternatively, by the complex shear modulus $G^{*}$, defined as

$$
G^{*} \equiv \frac{\hat{\imath}}{\hat{\varepsilon}}
$$

as a function of angular frequency $\omega$. Furthermore,

$$
\begin{aligned}
& \hat{\tau} \equiv \tau_{0} \exp (i \omega t+\phi) \\
& \hat{\varepsilon} \equiv \varepsilon_{0} \exp (i \omega t) \\
& \hat{\varepsilon} \equiv i \omega \hat{\varepsilon}
\end{aligned}
$$

in which $\phi$ is the phase angle.

$G^{*}$ and $\eta^{*}$ are decomposed into their real and imaginary parts according to

$$
G^{*} \equiv G^{\prime}(\omega)+i G^{\prime \prime}(\omega)
$$

and

$$
\eta^{*} \equiv \eta^{\prime}(\omega)-i \eta^{\prime \prime}(\omega)
$$

From equations (3) - (9) it follows:

$$
\begin{aligned}
& \eta^{\prime}=G^{\prime \prime} / \omega \\
& \eta^{\prime \prime}=G^{\prime} / \omega .
\end{aligned}
$$

$G^{\prime}(\omega)$ and $G^{\prime \prime}(\omega)$ are the so-called dynamical moduli, or, more specifically, $G^{\prime}(\omega)$ is called the storage modulus and $G^{\prime \prime}(\omega)$ the loss modulus. The moduli $G^{\prime}$ and $G^{\prime \prime}$ are interconnected by the mechanical analogue of the Kramers-Kronig relations, but for applying them one must know either $G^{\prime}$ or $G^{\prime \prime}$ completely as a function of $\omega[4,5]$. Usually, $G^{\prime}$ or $G^{\prime \prime}$ can be determined in one and the same experiment, albeit in a limited frequency range only.

From the principle of passivity general expressions for $G^{\prime}(\omega)$ and $G^{\prime \prime}(\omega)$ have been derived. For a material that cannot maintain a strain at constant shear, neither be strained instantaneously, these expressions read:

$$
\begin{aligned}
& G^{\prime}=\omega \eta^{\prime \prime}=\sum_{p=1}^{m} G_{p} \frac{\omega^{2} \tau_{p}^{2}}{1+\omega^{2} \tau_{p}^{2}} \\
& G^{\prime \prime}=\omega \eta^{\prime}=\omega \eta_{\infty}+\sum_{p=1}^{m} G_{p} \frac{\omega \tau_{p}}{1+\omega^{2} \tau_{p}^{2}}
\end{aligned}
$$

The quantities $G_{p}$ and $\tau_{p}$ are the relaxation strengths and the relaxation times, respectively, and $\eta_{\infty}$ is the infinite-frequency viscosity.

An equivalent description of linear viscoelasticity is provided by the complex compliance, $J^{*}$, defined as

$$
J^{*} \equiv \frac{\hat{\varepsilon}}{\hat{\tau}}
$$

and decomposed as

$$
J^{*}=J^{\prime}(\omega)-i J^{\prime \prime}(\omega) \text {. }
$$

For materials for which equations (12) and (13) are valid the general expression for $J^{\prime}$ and $J^{\prime \prime}$ read:

$$
J^{\prime}(\omega)=\sum_{k=1}^{m} J_{k} \frac{1}{1+\omega^{2} t_{k}^{2}}
$$

$$
J^{\prime \prime}(\omega)=\frac{1}{\omega \eta_{0}}+\sum_{k=1}^{m} J_{k} \frac{t_{k}}{1+\omega^{2} t_{k}^{2}}
$$

The quantities $J_{k}$ and $t_{k}$ are the so-called retardation strengths and retardation times and $\eta_{0}$ is the zerofrequency viscosity. Because of equations (4) and (14) $J^{*}=1 / G^{*}$, so that the sets of $(m+1)$ parameters occurring in the expressions for $G^{\prime}$ and $G^{\prime \prime}$ and in those for $J^{\prime}$ and $J^{\prime \prime}$ are interrelated. An important property is

$$
\tau_{p}>t_{p}(\text { all } p) .
$$

On the basis of certain assumptions about the molecular or microstructure of a system predictions about these parameter sets can sometimes be derived.

We will apply theories developed to describe the linear viscoelasticity of emulsions. Because $G^{\prime}$ and $G^{\prime \prime}$ 
or, equivalently, $\eta^{\prime}$ and $\eta^{\prime \prime}$ are the quantities primarily measured, the prediction will, when feasible, be cast in the general forms (12) and (13).

\section{First model of Oldroyd [2]}

The mentioned moduli can be measured straightforwardly, but there is no general way to derive from them the microstructure of a system. By developing a mathematical model from an assumed structure it is, however, possible to correlate measured and calculated moduli. In this way, one or more characteristic parameters of the system may be obtained.

In 1953 Oldroyd calculated the linear viscoelasticity of an emulsion consisting of spheres (radius a, volume fraction $\phi$ ) of one liquid (viscosity $\eta_{d}$ ) immersed in another liquid (viscosity $\eta_{s}$ ); the interfacial tension between the two liquids is $\gamma$. His result for $\eta^{*}$ reads:

$$
\eta^{*}=\eta_{0} \frac{1+i \omega t^{\prime}}{1+i \omega \tau}
$$

in which $t^{\prime}$ is a retardation time and $\tau$ is a relaxation time.

Recasting result (19) in the form of expressions (12) and (13), one gets (with $m=1$ ):

$$
\begin{aligned}
& \omega \eta^{\prime \prime}=G^{\prime}=\eta_{0} \frac{\tau-t^{\prime}}{t^{2}} \cdot \frac{\omega^{2} \tau^{2}}{1+\omega^{2} t^{2}} \\
& \omega \eta^{\prime}=G^{\prime \prime}=\eta_{0} \frac{t^{\prime}}{\tau} \omega+\eta \frac{\tau-t^{\prime}}{\tau^{2}} \cdot \frac{\omega \tau}{1+\omega^{2} \tau^{2}}
\end{aligned}
$$

Oldroyd succeeded in expressing the three quantities introduced in equation (19) into the system parameters; he found:

$$
\begin{aligned}
& \eta_{0}=\eta_{s}\left(1+\frac{\eta_{s}+\frac{5}{2} \eta_{d}}{\eta_{s}+\eta_{d}} \phi\right) \\
& t^{\prime}=A \frac{a}{\gamma}\left(B-12 \eta_{s} A \phi\right) \\
& \tau=A \frac{a}{\gamma}\left(B+8 \eta_{s} A \phi\right)
\end{aligned}
$$

in which

$$
\begin{aligned}
& A \equiv \frac{16 \eta_{s}+19 \eta_{d}}{40\left(\eta_{s}+\eta_{d}\right)} \\
& B \equiv 3 \eta_{s}+2 \eta_{d}
\end{aligned}
$$

As $t^{\prime}>0, \tau>0$, and $t^{\prime} / \tau<1$, it is obvious from equations (20) and (21) that $\eta^{\prime}$ as a function of frequency drops from a high level to a lower one, whereas $\eta^{\prime \prime}$ shows a maximum [6]. This maximum occurs for

$$
\omega_{\max }=\frac{1}{\tau} \approx \frac{\gamma}{a \eta_{s}} .
$$

There is a very simple physical interpretation of these results. At low frequencies the shear stresses are too weak to change noticeably the interface. Consequently, the viscosity is modified only by the extra dissipation due to the presence of spheres and the elasticity $\eta^{\prime \prime}$ is very small. At higher frequencies, however, the spheres deform as a consequence of the oscillating flow field, which results in an increase in the interfacial area. Because this is energetically unfavourable, energy is stored in the medium, which therefore shows elastic behaviour. At still higher frequencies the elasticity $G^{\prime}=\omega \eta^{\prime \prime}$ becomes constant, so that $\eta^{\prime \prime}$ shows a maximum. The angular frequency at which this occurs is equal to the inverse of the relaxation time; it therefore depends on the radii of the spheres, the viscosity of the continuous phase, and the interfacial tension acting along the surface.

\section{Second model of Oldroyd [3]}

In the first model it is assumed that the behaviour of the interface can be described by one single parameter, the interfacial tension. The possibility of dissipation of energy in the interface is not allowed for. If the interface is - and it certainly is for microemulsions an interfacial layer with a finite thickness, this might well be an oversimplification.

In his second model, Oldroyd allowed the interface to display an elastic as well as a viscous response, both on an area change and on a deformation. This can be formulated in the following rheological equation of state for the interfacial layer:

$$
\underline{P}=\{\gamma+(\varkappa+i \omega \sigma)(\operatorname{tr} \underline{E})\} \underline{1}+2(\mu+i \omega \zeta) \underline{E} .
$$

So, the relation between the surface-stress tensor $\underline{P}$ and the surface-strain tensor $E$ can be describe in terms of five parameters; the interfacial tension $\gamma$, the dynamic-area elasticity $\varkappa$ with corresponding viscosity $\sigma$, and the dynamic-shear elasticity $\mu$ with corresponding viscosity $\zeta$. In equation (28) tr $\underline{E}$ signifies the trace and $\bar{E}$ the traceless part of $E$.

Introduction of four additional parameters in the expression for $\eta^{*}$ appears to lead to one more 
relaxation time and one more retardation time or, equivalently, in the expression for $\eta^{\prime}$ and $\eta^{\prime \prime}$ of the type of equations (20) and (21), to one more relaxation mechanism.

\section{Oldroyd's second model at higher concentrations [7]}

Although Oldroyd's results contain terms of the order $\phi^{2}$ and higher, Oldroyd himself demonstrated that these terms cannot be correct. Deviations from linearity in $\phi$ may become serious above about $5 \%$. When reasonable values for $a, \eta_{d}, \eta_{s}$, and $\gamma$ are assumed, however, the maximum value of $\eta^{\prime \prime}$ comes out to be only a few percents of $\eta_{0}$. Measurements then become rather inaccurate. An extension of Oldroyd's results to higher $\phi$-values is needed if we wish to derive information about interfacial properties from viscoelastic measurements.

Such an extension was arrived at by using a cell model [7]. The general result for $\eta^{*}$, obtained with a symbolic computer program, can be written as

$$
\frac{\eta^{*}-\eta_{s}}{\eta_{s}}=\phi \frac{\alpha_{0}+\beta_{0} H+\gamma_{0} H^{2}}{\alpha_{0}^{\prime}+\beta_{0}^{\prime} H+\gamma_{0}^{\prime} H^{2}}
$$

where

$$
H=\frac{i a \eta_{s} \omega}{\gamma}
$$

The parameters $\alpha_{0}, \alpha_{0}^{\prime}, \beta_{0}, \beta_{0}^{\prime}, \gamma_{0}$, and $\gamma_{0}^{\prime}$ are related to the parameters of the previous sections. Because these relations are rather complex, the reader is referred to Appendix I of reference [7].

Our measurements of $\eta^{\prime}$ and $\eta^{\prime \prime}$ will be analyzed on the basis of equation (29).

\section{Experiments}

\section{Materials}

The non-ionic surfactant polyoxyethylene (6.8) nonylphenol (NNP 7) was supplied to us by Servo Co., Delden, The Netherlands. This material was for our purposes purified by extraction of polyethylene glycol with ethylacetate (Weibull method [8]). Nhexane was obtained from Baker Chemicals (p. a. grade) and was used as received, whereas the water was destilled three times.

\section{Phase diagram}

Bottles, about $40 \mathrm{ml}$ each, were filled with oil, water, and surfactant in a series of selected compositions and sealed afterwards. They were kept in water-thermostats, the temperatures of which had values between 0 and $40^{\circ} \mathrm{C}$ and varied less than 0.1 centigrade.
Usually, no observable changes took place any longer after 24 hours of standing, and a one-, two-, or three-phase system had formed.

For reasons to be explained later we were especially interested in the temperature-composition boundaries of the one-phase microemulsion region. By measuring, at a range of temperatures, the volumes of the phases in the two-phase region and extrapolating the overall composition to zero-volume of the non-microemulsion phase, the boundary point at that composition could be determined rather accurately.

\section{Rheological measurements}

Oscillatory shear measurements were carried out in the frequency range $3700-235000 \mathrm{~Hz}$ with a nickel tube $[9,10]$. This instrument essentially consists of a circularly magnetized Ni-tube which is set in torsional vibration. Resonance of the tube occurs at a discrete set of frequencies in the range mentioned. The liquid surrounding the tube has a profound influence on these resonances. The resonance frequency shifts to lower values and, furthermore, the resonance curve is broader, both relative to air. From the shift and the broadening the viscoelastic properties of the liquid can be obtained.

\section{Dynamic light scattering}

In the light-scattering apparatus we used a He-Ne laser of $10 \mathrm{~mW}$. The intensity of the scattered light (with a wavelength of $632.8 \mathrm{~nm}$ ) is detected with a photomultiplier, the output of which is coupled with a digital storage oscilloscope (Philips PM3310). This instrument measures the output of the photomultiplier at 256 discrete equidistant time events. These data are transmitted to a DEC LSI-11/02 microcomputer system that evaluates the autocorrelation function. This function is stored in memory and the oscilloscope performs another set of $\mathbf{2 5 6}$ measurements. The average of many correlations (usually 500) can be obtained in this way and the final autocorrelation function is plotted with a HP 7225 A plotter.

\section{Results and discussion}

\section{Analysis of the surfactant}

Although non-ionic surfactants are usually considered to form one component, they are in fact a mixture of different chemical compounds. The surfactant we used,

$$
\left.\mathrm{C}_{9} \mathrm{H}_{19}-\mathrm{O}-\mathrm{O}+\mathrm{CH}_{2}-\mathrm{CH}_{2}-\mathrm{O}\right)_{m} \mathrm{H}
$$

is a mixture of molecules with different values for $m$. The manufacturer stated an average value of 7 (therefore NNP 7).

This average, $\bar{m}$, can be determined by proton NMR spectroscopy. In the NMR spectrum the aliphatic, the aromatic, and the ethylene oxide hydrogen atoms give rise to signals at three distinct positions, centered at about 1, 7, and $4 \mathrm{ppm}$, respectively [11]. The spectrum of our surfactant is presented in figure 1 and from the integrated signal we calculated $\bar{m}=6.8$, 


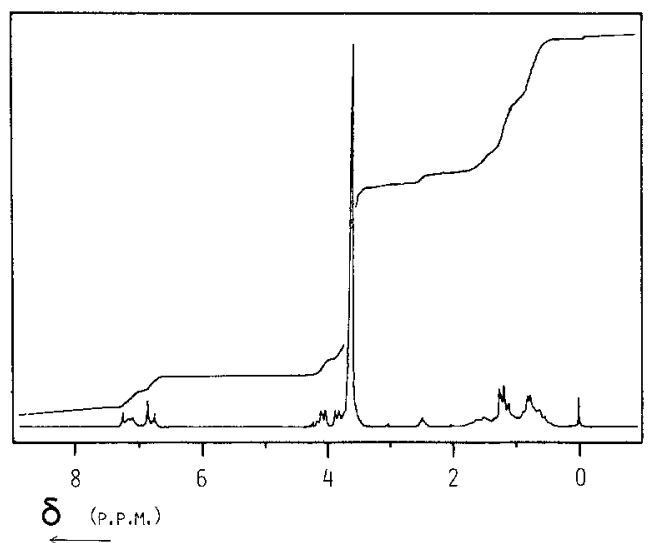

Fig. 1. Proton NMR spectrum of polyoxyethylene (6.8) nonylphenol (NNP7)

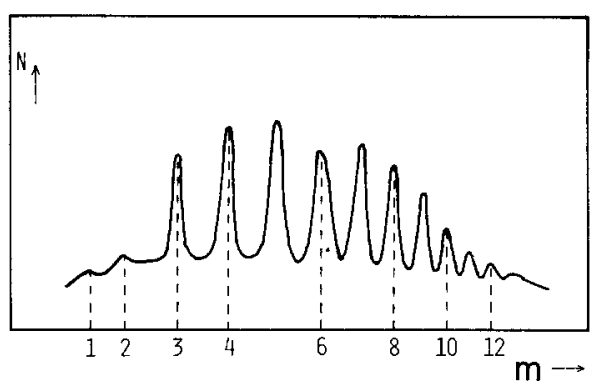

Fig. 2. HPLC curve of the polyoxyethylene (6.8) nonylphenol (NNP7). $m$ : number of ethyleneoxide units per molecule; $N$ : amount of surfactant with specified value of $m$

in good agreement with the specifications of the supplier.

Gradient elution HPLC was used for determining the distribution of the different compounds, according to Van der Maeden et al. [12]. The results of this techique are given in figure 2 . Hence it can be concluded that molecules with values of $m$ up to about twice the average are present in significant amounts.

So, it seems rather doubtful whether it is possible to treat the surfactant as a pseudo-component. Nevertheless, this is usually done and it seems to be justified judged from the measured cloud points of various samples of polydisperse surfactants with different values of $\bar{m}$ as given in figure 3 . So, a mixture of different homologous surfactants still has one sharp cloud point, which is determined by the average ethylene-oxide content. If more than one macroscopic phase is present, however, the distribution of the surfactant in the separate phases is usually modified strongly $[13,14]$. This was the main reason why we decided to restrict our experiments, for the time being,

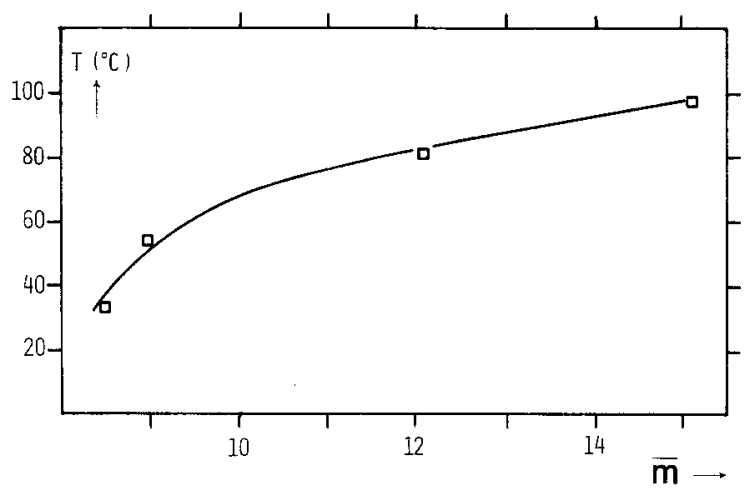

Fig. 3. Cloud points of various polyoxyethylene $(m)$ nonylphenols as a function of the average number of ethyleneoxide units per molecule $(\bar{m})$

to microemulsions in the one-phase region, the composition then being exactly known.

\section{Phase diagram}

The results concerning the phase behaviour of our systems are given in a form which resembles the presentation of Shinoda and Kuneida [15]. For a fixed surfactant concentration they gave the volumes of the different phases as a function of temperature and oil/ water composition. For waterrich systems they observed in a certain temperature range of one-phase system and for oil-rich systems the same. The former were oil-in-water $(\mathrm{o} / \mathrm{w})$ and the latter water-in-oil ( $\mathrm{w} /$ o) microemulsions. At intermediate compositions three-phase systems were observed in a certain temperature range.

In this paper the investigations are restricted to onephase oil-in-water microemulsions. The temperature traject of stability of these systems at the lower side is formed by the solubilization point and by the cloud point at the high temperature side. These points are given in figure 4 as a function of surfactant/oil ratio (by weight). The boundaries of the one-phase area are clearly given by two straight lines. From figure 4 it appears that the amount of water does not influence the range of stability of these systems in the indicated concentration range of the surfactant.

This last point can be readily accounted for. As oil we have taken $n$-hexane and this was done with a special purpose. It was found that for solubilizing $n$ hexane around room temperature a surfactant was required with an extrapolated cloud point below the freezing point of water (NNP 7, see fig. 3). This means that in these microemulsions the continuous phase consists of almost pure water. Since microemul- 


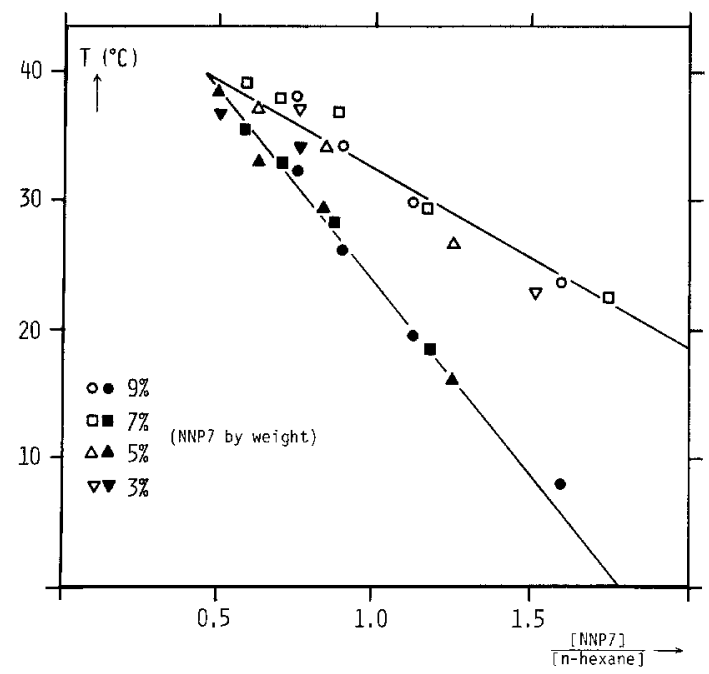

Fig. 4. Partial phase diagram for $n$-hexane/water/polyoxyethylene (6.8) nonylphenol microemulsions. The one-phase region is given as a function of ratio surfactant/oil (by weight)

sions are usually assumed to be dilutable with their continuous phase, this explains the observation made from figure 4.

\section{Rheological measurements for systems of spheres}

There is much debate on the structure of microemulsions. Especially in the three-phase region the nature of these systems is not clear. In the one-phase region, on the other hand, it is usually assumed that they are dispersions of spherical droplets. Our results show, however, that even for these systems the structure can be quite complex. There is a variety of methods for determining whether a system consists of a dispersions of spheres. The results of them will be given subsequently, but first the final picture that emerges from them will be presented. The region of the phase diagram where spheres occur is represented as the shaded area in figure 5 . As can be seen, this is only a minor part of the total diagram.

The first indication for the occurrence of spheres we have obtained from the value of $\eta^{\prime}$ at the lowest frequency. If at this frequency $(f=3700 \mathrm{~Hz})$ the system shows no elastic effects (which will later be shown to be theoretically correct for spheres), this value can be equated to the stationary viscosity. There is a rather famous relation, first derived by Einstein, between the viscosity of a dispersion of hard spheres, $\eta$, the volume fraction of the dispersed phase, $\phi$, and the viscosity of the solvent, $\eta_{s}[16,17]$. It reads:

$$
\frac{\eta}{\eta_{s}}=1+\frac{5}{2} \phi
$$

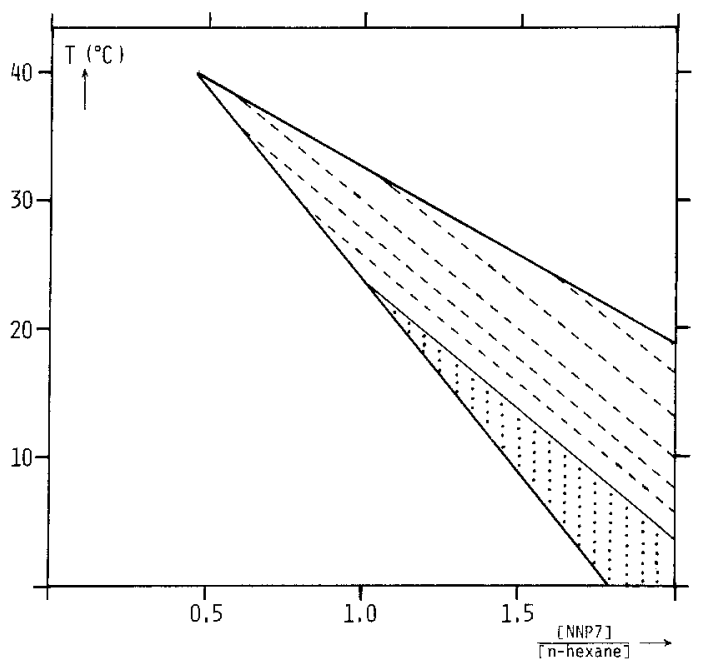

Fig. 5. Partial phase diagram for $n$-hexane/water/polyoxyethylene (6.8) nonylphenol microemulsion (NNP7), with the sphere region $(:)$ and the region with unusually high elastic properties $(==)$

This equation is only valid at very low concentrations, because interparticle effects are not taken into account. At the moment, there is no rigorous method developed to provide for this. Simha, however, presented a semi-empirical approach based on a cell model $[18,19]$. In this model each sphere is thought to be contained in a cell, and in a pure cell model the volume fraction of the sphere in this cell is the same as the overall volume fraction of spheres in the system. Solving the hydrodynamic equations, Simha derived:

$$
\frac{\eta}{\eta_{s}}=1+\frac{10 \phi R^{3}\left(R^{7}-1\right)}{4\left(R^{10}+1\right)-25\left(R^{7}+R^{3}\right)+42 R^{5}},
$$

with

$$
R=f \phi^{-\frac{1}{3}} .
$$

In addition to the volume fraction $\phi$, this expression contains a factor $f$. A value of 1 for this parameter means that the viscous behaviour of the system can be described by a pure cell model. Different values for $f$ are often obtained and this is usually attributed to attractive forces between the particles. Thomas, by analysing many experimental results, concluded that a value of 1.111 is usually found [20].

From figure 6 it is clear that our results correspond quite well to $f=1$ if the volume fraction of the dispersed phase is calculated from the volumed of the three components as

$$
\phi=\frac{V_{\text {oil }}+V_{\text {surf }}}{V_{\text {oil }}+V_{\text {surf }}+V_{\text {water }}} .
$$




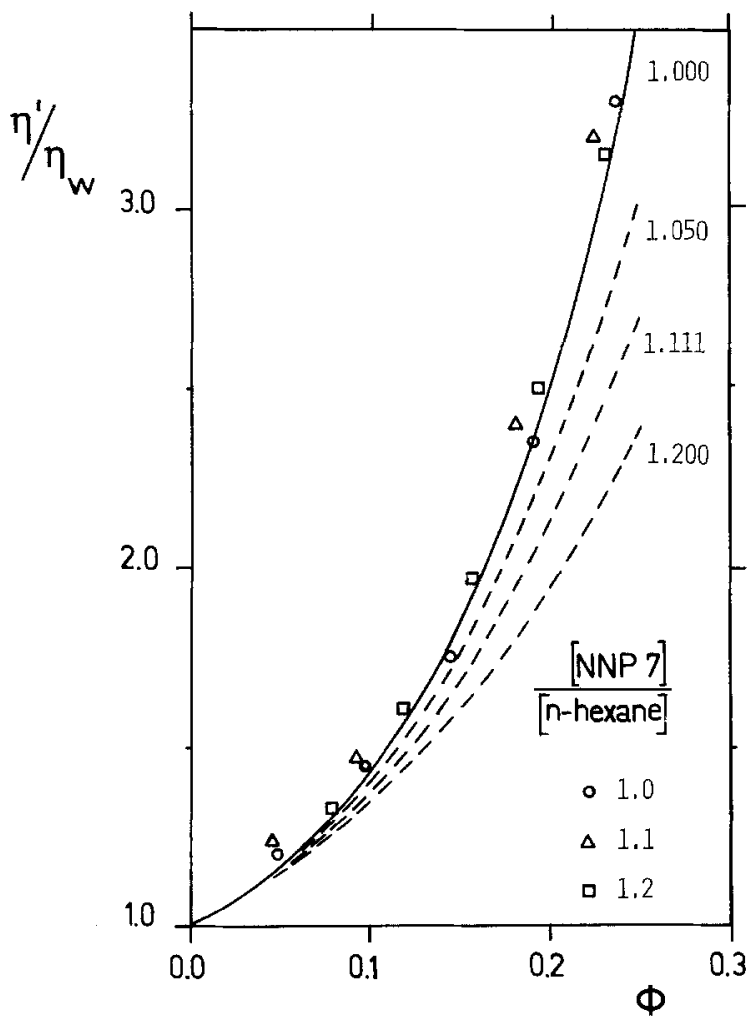

Fig. 6. Dynamic viscosity $\eta^{\prime}$ relative to the viscosity of water $\left(\eta_{w}\right)$ at $3700 \mathrm{~Hz}$ as a function of the volume fraction of the dispersed phase $(\phi)$ for various concentration ratio's surfactant/oil. The solid line is calculated according to Simha's formula with $f=1$. Calculated lines for other values of $f$ are given as broken lines

We consider this a strong indication that the microemulsions under these circumstances are suspensions of spherical droplets. It is true, however, that the hydrodynamically effective volume fraction may exceed a little bit a value of $\phi$ as given by equation (34) as a consequence of partial hydratation of the detergent layer. This effect is small, as will be discussed later on.

It must be emphasized, however, that Einstein's and Simha's fomula were derived for a system of hard spheres. Taylor obtained a formula for the viscosity of a dispersion of liquid spheres into another liquid. His formula is [21]:

$$
\frac{\eta}{\eta_{s}}=1+\phi\left(\frac{\eta_{s}+\frac{5}{2} \eta_{d}}{\eta_{s}+\eta_{d}}\right) .
$$

This expression is clearly an extension of the one of Einstein. For $n$-hexane dispersed in water (without a surfactant) it reduces to approximately

$$
\frac{\eta}{\eta_{s}}=1+1.35 \times \phi .
$$

Apparently, this formula predicts substantially lower viscosities than predicted by equation (31). Our results in the low-volume-fraction region correspond better to Einstein's formula. To explain this, one must go back to the starting assumption of Taylor. He derived his formula by solving the hydrodynamic equations. But he had to assume some boundary conditions and by doing so he assumed that momentum transport between the dispersed and the continuous phase is not hindered by the interface. This means that the surfactant layer around the oil droplets is a rather stiff or viscous shell.

The first indication for the existence of spheres stems from viscosity measurements, the second one comes from dynamic light scattering. With this technique it is possible to determine the diffusion coefficient of particles in a dispersion from the intensity autocorrelation function of the scattered light. One can derive [22]:

$$
g^{(2)}(q, t)=1+\exp \left(-2 q^{2} D t\right)
$$

with

$$
q=\frac{4 \pi n}{\lambda} \sin \frac{\theta}{2}
$$

where $g^{(2)}(q, t)$ is the normalized homodyne autocorrelation function, $n$ is the refractive index of the continuous phase, $\theta$ is the scattering angle, $\lambda$ is the wavelength of the laser light in vacuum, and $D$ is the diffusion coefficient.

If the particles are assumed to be spheres of uniform radius $a$, the Stokes-Einstein relation gives this parameter in terms of measurable quantities:

$$
a=\frac{k T}{6 \pi \eta_{s} D},
$$

in which $k$ is Boltzmann's constant and $T$ is the absolute temperature. But also for structures other than spheres the autocorrelation function can still be an exponential function, and by applying the StokesEinstein relation, one gets a value for the radius. So, without further knowledge about the system under consideration it is not clear whether this value is reliable.

This knowledge can be obtained from data about the surfactant that we found in the literature. It is a well-known fact that surface-active compounds at flat water/air surfaces from a monomolecular layer [23]. At very low values of the surface tension all surfactant molecules occupy the same area. For polyoxyethylene 
$(m)$ nonylphenols this area is known as a function of $m[24,25]$ :

$$
A_{\text {surf }}=18 \sqrt{m} \text {, }
$$

in which $A$ is obtained in $\AA^{2}$. If $m=6.8$, this formula yields :

$$
A_{\mathrm{NNP}}=47 \AA^{2} .
$$

As mentioned earlier, we have chosen this surfactant because it is almost insoluble in both water and $n$ hexane at room temperature. This implies that in these microemulsions all surfactant molecules are located at the interface. If we assume further that the system contains spheres of uniform radius, we can calculate the total interfacial area if the amount of surfactant is known [26]. If the amount of oil is also known, this gives for the radius of spheres:

$$
a=\frac{3 M_{\text {surf }}}{1000 N_{A} \varrho_{\text {oil }}} \cdot \frac{g_{\text {oil }}}{g_{\text {surf }} \cdot A_{\text {surf }}} .
$$

where $M_{\text {surf }}$ is the molecular weight of the surfactant, $N_{A}$ is Avogadro's number, $\varrho_{\text {oil }}$ is the density of $n$ hexane, $g_{\text {surf }}$ is the weight fraction of surfactant, $g_{\text {oil }}$ is the weight fraction of $n$-hexane, and $A_{\text {surf }}$ is the molecular area of the surfactant.

This formula gives the radius of the spheres as a function of, among other parameters, the weight ratio surfactant-to-oil. We have determined the hydrodynamic radius of these particles by dynamic light scattering in the sphere region at surfactant-to-oil ratios of 1.0 and 1.2. As can be seen from table 1, we found values for $a$ of 120 and $100 \AA$, respectively. These values were obtained for $\phi=0.1$ just above the solubulization temperature at that composition and were calculated with equation (39). For $\eta_{s}$ we used the viscosity of water at that particular temperature. If one takes into account that approximately one third of the

Table 1. Interfacial parameters and radius of the spheres

\begin{tabular}{lccccccc}
\hline & \multicolumn{2}{c}{ I } & \multicolumn{2}{c}{ II } & \multicolumn{2}{c}{ III } \\
\hline$g_{\text {surf }} / g_{\text {oil }}$ & 1.0 & 1.2 & 1.0 & 1.2 & 1.0 & 1.2 \\
$\gamma[\mathrm{mN} / \mathrm{m}]$ & 0.06 & 0.08 & 0.06 & 0.08 & 0.06 & 0.08 \\
$S$ & 7.0 & 7.0 & 7.0 & 7.0 & 7.0 & 7.0 \\
$Z$ & 25.0 & 25.0 & 25.0 & 25.0 & 25.0 & 25.0 \\
$2 M+3 K$ & 0 & 0 & 0.45 & 0.45 & $\geqslant 1$ & $\geqslant 1$ \\
$F$ & 1.05 & 1.06 & 1.03 & 1.04 & 1.05 & 1.06 \\
$\left.a^{2}\right)[\AA]$ & 120 & 100 & 120 & 100 & 120 & 100 \\
\hline
\end{tabular}

a) obtained by dynamic light scattering. surfactants molecule is situated in the oil phase (so that the actual weight fraction of the oil is increased by a third of the surfactantweight fraction), equation (41) yields with $M=519$ at $g_{\text {surf }} / g_{\text {oil }}=1.0$ and 1.2 , respectively:

$$
a=105 \AA \text { and } a=85 \AA \text {. }
$$

This is the distance from the centre of the sphere to that part of the interfacial layer where the hydrophobic and hydrophilic parts of the surfactant are attached to each other. To be able to compare the calculated with the measured hydrodynamic radius one has to take into account the conformation of the polyethyleneoxide chains in the water phase. Information about this can be obtained from the simple formula (40). If these chains would lay along the interface, $A_{\text {surf }}$ would be a linear function of $m$, and if the chains were oriented perpendicular to the surface, $A_{\text {surf }}$ would be independent of $m$. Obviously, neither of these possibilities does apply. In fact, equation (40) can be explained if the polyethyleneoxide chains form coils that partly occupy the surface. This means that the microemulsion droplets for $g_{\text {surf }} / g_{\text {oil }}=1.0$ look like the graphical presentation as given in figure 7 . As the real radius of the spheres is about 10 to $20 \AA$ larger than the one calculated with equation (41), the agreement between this radius and the hydrodynamic radius obtained from dynamic light scattering is rather good. This is the second indication that in the shaded area in figure 5 these microemulsions contain spherical oil droplets.

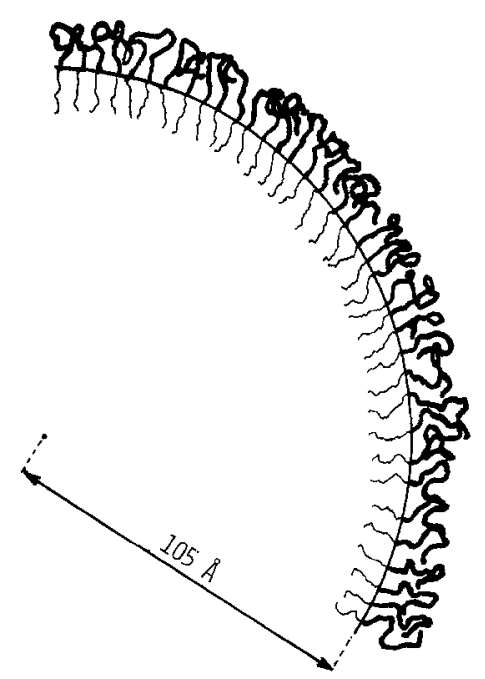

Fig. 7. Model of the dispersed phase in the sphere region (drawn to scale) 


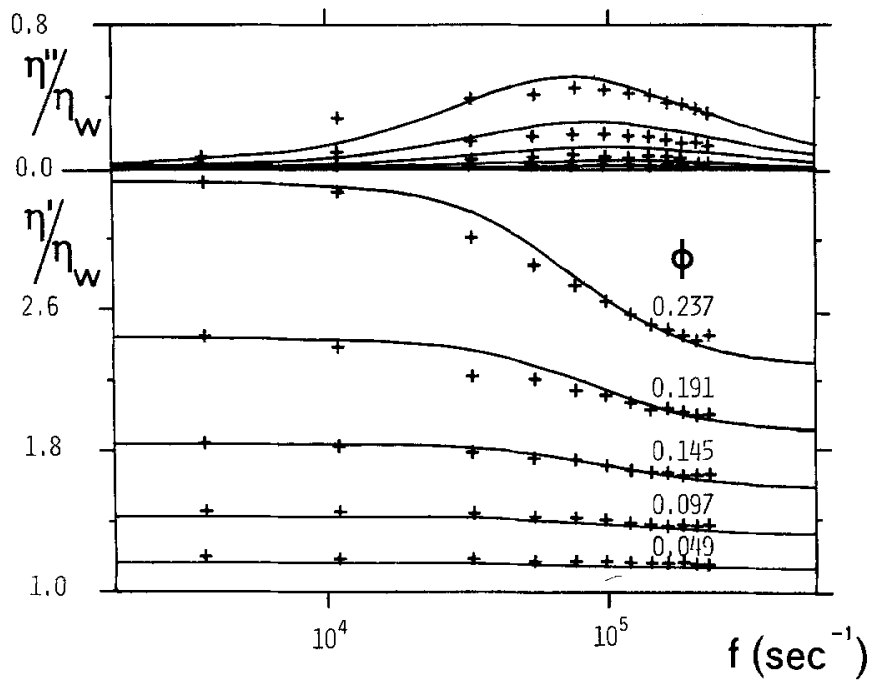

Fig. 8. Dynamic viscosities $\eta^{\prime}$ and $\eta^{\prime \prime}$ (relative to the viscosity of water) as a function of frequency $(f)$ for various values of the volume fraction of the dispersed phase $(\phi)$. Ratio surfactant/oil: 1.0 (by weight). Temperature: $23.0^{\circ} \mathrm{C}$. Solid lines: fitted to experimental results with Oldroyd's second model

The third and last indication for the existence of spheres comes from the viscoelastic measurements themselves. Both models of Oldroyd take the existence of spheres as a starting point and both predict that $\eta^{\prime \prime}$ as a function of frequency shows (at least) one maximum. For values usually assumed to be correct for $a$ and $\gamma$ in microemulsions this maximum must occur in the $\mathrm{kHz}$ range. As an example we have presented some of our measurements in figure 8 and hence it can be concluded that the maximum in $\eta^{\prime \prime}$ and the drop in $\eta^{\prime}$ do occur in the required frequency range.

We now come to the central theme of this paper, the interpretation of the viscoelastic measurements in terms of the models of Oldroyd. The first model, however, cannot explain all measurements. Nevertheless, it predicts very well the transition in the proper range. But this model has the great advantage that it contains only one adjustable parameter, the interfacial tension $\gamma$, provided that the radius of the spheres and the viscosity of the continuous phase are independently determined (the former was obtained by dynamic light scattering and the latter followed from the composition of the continuous phase and was derived from the phase diagram). As already stated, Oldroyd's second model contains four additional parameters to account for the viscous and elastic properties of the interface. They can be made dimensionless by the interfacial tension, the radius of spheres, and the viscosity of the continuous phase:

$$
\begin{aligned}
& M=\frac{\mu}{\gamma} \\
& K=\frac{x}{\gamma} \\
& Z=\frac{\zeta}{a \eta_{s}} \\
& S=\frac{\sigma}{a \eta_{s}} .
\end{aligned}
$$

With these additional parameters the model predicts, in general, two transitions. We have observed only one. From the values of $\eta^{\prime}$ at $3700 \mathrm{~Hz}$ we can conclude that at this frequency the systems behave as a dispersion of hard spheres. (This means that $\eta^{\prime \prime}$ $(3700)=0$. For $\phi<0.2$ this is correct. For higher values of $\phi$, however, one observes that the $\eta^{\prime \prime}$ curve has shifted to lower frequencies as a consequence of hydrodynamic interaction, so that these systems show some elasticity at the mentioned frequency. For these high $\phi$ values the elastic effects vanish at lower frequencies, as can be seen from the fitted upper curve in fig. 8.) A transition at lower frequencies than the range covered by the nickel tube can be rules out, as under stationary conditions (as determined by dynamic light scattering) the results are still constant with a sphere model. This means that our results can be interpreted in three different ways:

I. It can be shown [28] that one of the transitions vanish if

$$
M=0 \text { and } K=0 \text {, }
$$

which means that the interface shows no elastic effects and, consequently, behaves as a twodimensional Newtonian liquid.

II. Both transitions occur at the same frequency, which implies that $\mu$ and $x$ are on the order of $\gamma$, or

$$
M \text { and/or } K \approx 1 .
$$

III. One of the transitions occurs at very high frequencies. This would mean that the elastic properties of the interface are very strong, or

$$
M \text { and/or } K \gg 1 \text {. }
$$

By fitting the parameters of Oldroyd's second model, using equation (29), which is also valid at higher concentrations, we obtained three sets of values for the parameters. They are given in table 1 . The most important point that can be observed from it is that all 
three interpretations yield the same value for the interfacial tension $\gamma$.

In table $1 F$ is the factor with which the volume fraction of the dispersed phase has to be multiplied in order to obtain a good fit. This parameter, therefore, gives an indication about the amount of water that is hydrated by the polyethyleneoxide chains. It must be emphasized, however, that $f$ (from Simha's formula) and $F$ cannot be determined independently from the results presented here. We have chosen $f=1.0$ and have fitted $F$ with the results and values obtained for $F>1.0$. This parameter has to be in that range, for hydration is a necessity for the thermodynamic stability of non-ionic microemulsions.

The solid lines in figure 8 have been calculated with the values for the parameters obtained in interpretation I. The other interpretations yield similar lines. From this figure two conclusions can be drawn:

i) At low frequencies $\eta^{\prime}$ is predicted very well by the model. With increasing frequency $\eta^{\prime}$ drops to lower values, but this effect is less abrupt than the model requires. A possible explanation is that the spheres are not of uniform size.

ii) As a function of frequency $\eta^{\prime \prime}$ shows the expected maximum. It is too broad, however, again indicating a distribution of spheres of different size. Furthermore, the measured and the theoretical concentration dependence of $\eta^{\prime \prime}$ do not correspond very well with each other. This can be explained by the fact that with the present equipment it is impossible to measure $\eta^{\prime \prime}$ as accurately as $\eta^{\prime}$. For this reason, only the values for $\eta^{\prime}$ were used in the fitting procedure.

Finally, we focus our attention on the theoretically and practically most important parameter of all, the interfacial tension $\gamma$. At first sight, the values we found for this parameter (see table 1) may seem somewhat high compared to the often mentioned value of $10^{-3} \mathrm{mN} / \mathrm{m}$. But one must take into account that this last value refers to the interfacial tension between flat water $/$ m.e. or oil $/$ m.e. interfaces. And as already stated, we restricted our investigations to macroscopic one-phase systems. This can be illustrated by referring to the work of Saito and Shinoda [27].

They have measured the interfacial tension between macroscopic phases in two- and three-phase non-ionic microemulsions (slightly different from our system). It is not clear from the onset whether their $\gamma$ may be equated to ours, but, nevertheless, they are of the same order of magnitude. These authors measured values ranging from 0.2 to $0.001 \mathrm{mN} / \mathrm{m}$.

The latter value is that for three-phase systems. The closer to this region, the lower the interfacial tension.

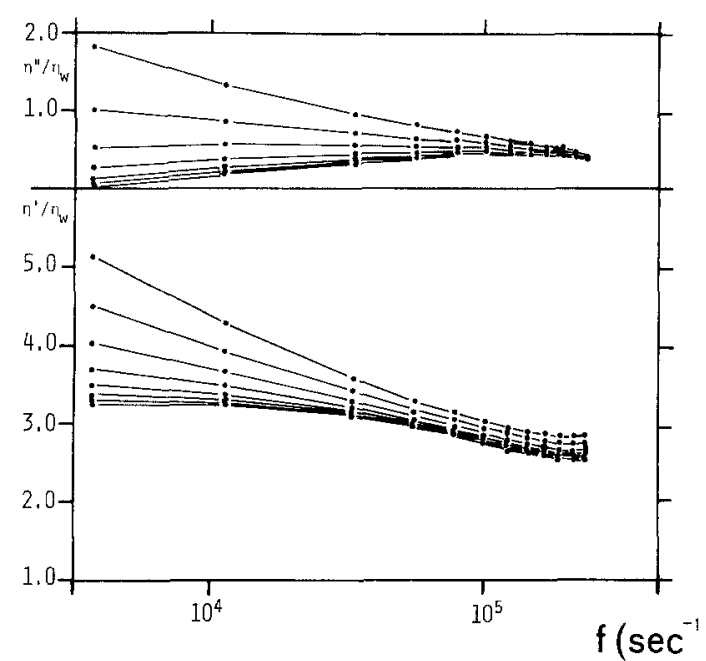

Fig. 9. Dynamic viscosities $\eta^{\prime}$ and $\eta^{\prime \prime}$ (relative to the viscosity of water) as a function of frequency $(f)$. Volume fraction $\phi=0.231$. Ratio surfactant/oil: 1.2 (by weight). Both viscosities increase with temperature. Measurements were carried out at 18.27, 18.76, 19.21, $19.75,20.48,21.35,22.43$, and $23.83^{\circ} \mathrm{C}$

And this is exactly the trend we found, for the system with $g_{\text {surf }} / g_{\text {oil }}=1.0$ is closer to the three-phase region than the system with the other composition.

\section{Rheological measurements for systems with a more complicated microstructure}

With rising temperature the dynamic viscosities show a large increase, which means that a complex structure develops under these circumstances. This is illustrated in figure 9 for a microemulsion with $g_{\text {surf }} /$ $g_{\text {oil }}=1.2$ and $\phi=0.231$. The viscosities in this figure are expressed relative to the viscosity of water of the same temperature, which means that the ordinary temperature dependence of the viscosity of the continuous phase is eliminated. So, figure 9 is a direct manifestation of a changing structure in the system.

At rather low temperatures (only slightly above the solubilization temperature) we recognize the pattern indicative for spheres. By referring to figure 8 it can be seen that because of a different interfacial tension and a different radius for the spheres the maximum for $\eta^{\prime \prime}$ occurs at a higher frequency.

By increasing the temperature it is obvious from figure 9 that both viscosities attain higher values. Especially the change in the elastic effect $\left(\eta^{\prime \prime}\right)$ is quite remarkable. In the sphere region the system shows no elastic properties at the lowest frequency, whereas with rising temperature there is a dramatic increase of 


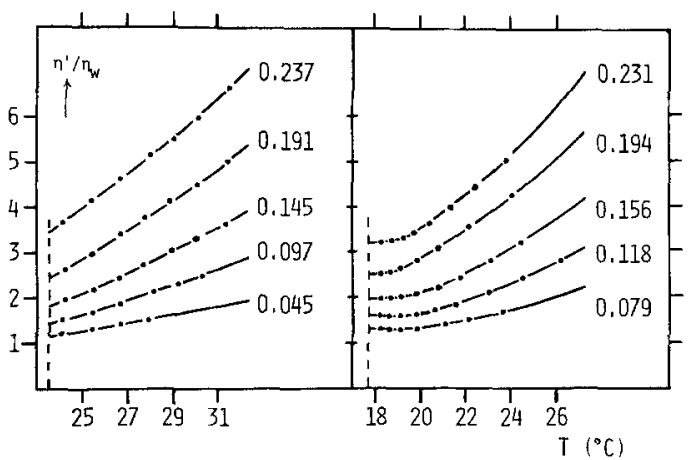

Fig. 10. Dynamic viscosity $\eta^{\prime}$ for $3700 \mathrm{~Hz}$ as a function of temperature (relative to the viscosity of water of the same temperature, $\eta_{w}$ ) for various volume fractions $\phi$. Surfactant/oil ratio is 1.0 (left) and 1.2 (right). Dashed line: solubilization temperature

$\eta^{\prime \prime}$. This means that new and larger relaxation times of the system emerge, indicating the formation of larger structures under these circumstances. These results are, however, not unique for this system. Measurements on cyclohexane/NNP 7/water microemulsions show qualitatively the same trend as a function of temperature [28].

The effect of temperature on the rheological properties (and, therefore, on the microstructure) is presented in a different way in figure 10 , where $\eta^{\prime} / \eta_{s}$ at $f=3700 \mathrm{~Hz}$ is given as a function of temperature. At $g_{\text {surf }} / g_{\text {oil }}=1.0$ it is clear that immediately above the solubilization temperature $\left(T_{s}\right)$ the dynamic viscosity increases. As at $T_{s} \eta^{\prime}(3700)$ corresponds very well with Simha's formula (see fig. 6), we conclude that only at this temperature there is a system of spheres. From the graphs for $g_{\text {surf }} / g_{\text {oil }}=1.2$ it follows that there is quite a temperature traject along which rheological properties are constant. As $\eta^{\prime}$ in this range is consistent with Simha's formula, the oil in this temperature range is dispersed as spherical droplets in water.

At the lowest concentration, see figure 10, at the right, the temperature traject where spheres are present is more than two centigrades, whereas at the highest volume fraction this traject is reduced to about one centigrade. This means that there is a weak concentration dependence of the temperature where the structure of the system changes.

Thus, we have demonstrated some drastic effects. But, unfortunately, it is not possible to derive the structure of a system from rheological measurements alone. We can only conclude from them at this moment that the length scale of the structure increases with rising temperature. Similar phenomena for sur- factant solutions have been known a long time. These effects are often attributed to a transition from spherical micelles to rods or cylinders [29]. Also viscoelastic properties of these systems are mentioned, mostly in connection with results of other techniques, such as NMR, dynamic light scattering, electric birefringence, and others $[30,31]$.

However, the transition from spheres to cylinders alone is not able to account for these strong elastic effects. It is absolutely necessary to take strong interactions between these cylinders into account. With such advanced rheological theories as put forward by Doi and Edwards it should be possible to account for these effects $[32,33]$. At the moment, further investigations are carried out on this subject; our attention is especially focussed on the transition from spheres to non-spherical structure.

\section{Conclusions}

About the measurements mentioned so far we may state that the rheological models of Oldroyd apply reasonably well to our non-ionic microemulsions in the sphere region. The most important parameter that can be obtained in this way is the interfacial tension between the continuous and the dispersed phase. The values for this parameter we obtained are in the same order of magnitude and show the same trend as the values for the interfacial tension between macroscopic phases reported by Saito and Shinoda.

Furthermore, other properties of the interfacial layer may be obtained in this way. There are, however, three different interpretations of our measurements, all yielding the same value for the interfacial tension. But the other properties of the interface may range from purely viscous to highly elastic.

Increasing the temperature of our microemulsions usually results in systems with very strong elastic effects. They show resemblance to viscoelastic surfactant solutions; so, it seems likely that a fluctuating network of oil-swollen micelles develops under these conditions.

\section{Acknowledgements}

The authors are grateful to Dr. ir. P. M. van der Velden and Drs. W. Jansen of the Chemische Fabriek Servo BV (Delden, The Netherlands) for the supply and further purification of the surfactant NNP 7. The assistance of the Analytical Chemical Department with the analysis of the surfactant is gratefully acknowledged and thanks are due to Prof. Dr. P. F. Mijnlieff for his valuable discussions of this paper. 


\section{References}

1. Lindman B, Danielson I (1981) Colloids and Surfaces $3: 391$

2. Oldroyd JG (1953) Proc Roy Soc London, Ser A 218:122

3. Oldroyd JG (1955) Proc Roy Soc London, Ser A 232:567

4. Ferry JD (1981) Viscoelastic Properties of Polymers, Wiley, New York

5. Bird RB, Armstrong RC, Hassager $O$ (1977) Dynamics of Polymeric Liquids, vol 1, Wiley, New York

6. Axelrod DR (1970) Adv in Mol Relaxation Processes 2:41

7. Oosterbroek M, Mellema J (1981) J Coll Interf Sci $84: 14$

8. Riesewijk G, Leeferink J (1973) Tenside Detergents 10:57

9. Waterman HA, Oosterbroek M, Beukema GJ, Altena EG (1979) Rheol Acta 18:585

10. Oosterbroek M, Waterman HA, Wiseall SS, Altena EG, Mellema J, Kip GAM (1980) Rheol Acta 19:497

11. Flanagan PW, Greff RA, Smith HF (1963) Anal Chem $35: 1283$

12. Van der Maeden FPB, Biermond MEF, Janssen PCGM (1978) J Chromatogr 149:539

13. Eshuis A, unpublished results

14. Smith DH (1983) paper presented at the 57th Colloid and Surface Science Symposium, Toronto

15. Shinoda K, Kuneida $H$ (1973) J Coll Interf Sci $42: 381$

16. Einstein A (1906) Ann Phys 19:298

17. Einstein A (1911) Ann Phys $34: 591$

18. Simha R (1952) J Appl Phys 23:1020

19. Frisch HL, Simha R (1956) in "Rheology, vol 1" (Eirich, ed), Academic Press, New York, p 525

20. Thomas DG (1965) J Coll Sci 20:267

21. Batchelor GK (1970) An Introduction to Fluid Dynamics, Cambridge University Press
22. Berne BJ, Pecora R (1976) Dynamic Light Scattering, Wiley, New York

23. Lange H (1966) in: "Nonionic Surfactants, vol 1" (Schick, ed), Marcel Dekker, New York

24. Hsiao L, Dunning HN, Lorenz PB (1956) J Phys Chem 60:657

25. Van Voorst Vader F (1960) Trans Faraday Soc 56:1078

26. Van Voorst Vader F, private communication

27. Saito H, Shinoda K (1970) J Coll Interf Sci 32:647

28. Oosterbroek M, Mellema J, Lopulissa JS (1981) J Colloid Interface Sci $84: 27$

29. Nagarajan R, Shah KM, Hammond S (1982) Coll and Surf $4: 147$

30. Ulmius J, Wennerström $H$, Johansson LBA, Lindblom $G$, Grarsholt S (1979) J Phys Chem 83:2232

31. Hoffmann H, Platz G, Rehage H, Schorr W, Ulbricht W (1981) Ber Bunsenges Phys Chem 85:255

32. Doi M, Edwards SF (1978) JCS Faraday II 74:560

33. Doi M, Edwards SF (1978) JCS Faraday II $74: 918$

Received September 22, 1983 ; accepted October 20, 1983

Authors' address :

A. Eshuis, J. Mellema

Department of Applied Physics

Twente University of Technology

P.O. Box 217

7500 AE Enschede, The Netherlands 